\title{
Avaliação da satisfação de usuários da Atenção Básica portadores de hipertensão e diabetes
}

\author{
Mirelle de Oliveria Saez, Luiz Augusto Facchini, Elaine Tomasi
}

\section{RESUMO}

Motivação: Este estudo tem como objetivo identificar a satisfação dos usuários hipertensos e diabéticos com o cuidado recebido na atenção básica.

Método: Análise descritiva e modelos hierárquicos aplicados à dados oriundos da avaliação externa do 2o ciclo do Programa de Melhoria do Acesso e da Qualidade da Atenção Básica ciclo II - PMAQ.

Resultados: Dos 47.927 usuários hipertensos e/ou diabéticos do estudo, observou-se que $80,3 \%$ (IC95\% 79,9-80,6) encontravam-se satisfeitos com o cuidado recebido. Após análise ajustada, o principal fator que afetou o desfecho foi a percepção negativa sobre o cuidado clínica recebido, reduzindo em $17,0 \%$ (IC95\% 0,82-0,84) a satisfação dos usuários.

Conclusões: Os resultados reforçam a relevância do investimento em ações voltadas para equipe de saúde, propiciam condições para melhoria na oferta do cuidado oferecido.

Palavras-chave: Atenção primária à Saúde; Satisfação do Paciente; Hipertensão; Diabetes Mellitus; Saúde Pública.

Revista da Rede APS 2019

Publicada em: 17/12/2019

DOI:10.14295/aps.v1i3.49

Mirelle de Oliveria Saez (Faculdade de Medicina, Universidade Federal do Rio Grande, Rio Grande, Rio Grande do Sul, Brasil)

Luiz Augusto Facchini (Universidade Federal de Pelotas, Pelotas, Rio Grande do Sul, Brasil)

Elaine Tomasi

(Universidade Federal de Pelotas, Pelotas, Rio Grande do Sul, Brasil)

Correspondência para: Mirelle de Oliveria Saez mirelleosaes@gmail.com 


\section{INTRODUÇÃO}

A atenção básica é porta de entrada no sistema de saúde brasileiro, e caracteriza-se como centro de comunicação da rede de atenção à saúde, devendo garantir acesso gratuito, integral e de qualidade aos serviços e cuidados em saúde (1). Nos últimos 30 anos, a avaliação tem evidenciado os avanços e lacunas no desenvolvimento do Sistema Único Saúde (SUS) e da Estratégia Saúde da Família (ESF), subsidiando a formulação de políticas e a resposta dos serviços (1). Desde 2011, o PMAQ - Programa de Melhoria do Acesso e da Qualidade da Atenção Básica, que completou seu terceiro ciclo em 2018, institucionalizou incentivo financeiro do Ministério da Saúde à melhoria do padrão da assistência oferecida aos usuários do SUS por meio das equipes de Saúde da Família (2).

Dentre os componentes fundamentais para a avaliação da qualidade dos serviços de saúde destaca-se a satisfação dos usuários, importante para medir resultados, reorganizar serviços e readequar ações (3-4). Considerando que o resultado do cuidado depende da efetiva participação de seus usuários, é imprescindível que estes também estejam envolvidos no processo avaliativo. A satisfação dos usuários com o serviço interferirá na adesão aos tratamentos, criação de vínculos, horizontalidade do cuidado e na adequação no uso dos recursos assistenciais $(3,5)$.

Estudos indicam que as pesquisas de satisfação de usuários podem resultar em melhoria na qualidade do atendimento de saúde. A divulgação de resultados de pesquisas de satisfação dos usuários é capaz de induzir gestores e profissionais de saúde a melhorar os serviços e ações de saúde 6 .

Estudos realizados na atenção primária à saúde referem uma prevalência de satisfação dos usuários em torno de $50 \%$ na Espanha e Finlândia, enquanto no Brasil, foi cerca de 70\% (7-9). Entretanto, investigações brasileiras sinalizam a insatisfação dos usuários com os horários de atendimento, o agendamento de consultas, a organização do serviço, a deficiência na estrutura e os encaminhamentos às especialidades (9-11). Além disso, na comparação com usuários de países da
América Latina e Caribe, observa-se que os brasileiros estão entre os mais insatisfeitos com o desempenho da saúde e com a qualidade do cuidado recebido (12).

Não foram encontrados estudos de satisfação direcionados a usuários com hipertensão (HAS) e diabetes (DM). Somente um estudo avaliou a satisfação dos pacientes hipertensos, sem incluir os diabéticos 5. A HAS e o DM são as doenças crônicas mais prevalentes na atenção básica, atingindo cerca de $20 \%$ e $8 \%$ da população, respectivamente, e constituem os principais fatores de risco para doenças cardiovasculares, das quais aproximadamente $80 \%$ são tratáveis na rede básica de saúde 13. Por este motivo, uma das ações dos serviços de atenção básica preconizadas pelo Ministério da Saúde é vincular os portadores de HAS e DM, garantindo tratamento e acompanhamento de qualidade, o que pode ser potencializado com usuários satisfeitos com o cuidado $(13,14)$.

Este estudo teve o objetivo de identificar a satisfação dos usuários hipertensos e diabéticos com o cuidado recebido na atenção básica, considerada um indicador da qualidade dos serviços de saúde. Os resultados do estudo podem auxiliar no planejamento e reorganização do processo de trabalho, propiciando avanços na qualidade do cuidado prestado na rede básica de saúde do SUS.

\section{Metodologia}

Neste estudo foram utilizados os dados oriundos da avaliação externa do 2 o ciclo do Programa de Melhoria do Acesso e da Qualidade da Atenção Básica ciclo II - PMAQ, cuja coleta de dados foi realizada de novembro/2013 a março/2014. O PMAQ trata da mais recente iniciativa do governo federal brasileiro de avaliação dos serviços de atenção básica, instituída a partir da portaria 1.654 de 19 de abril de 2011, com o intuito de ampliar o acesso e melhorar a qualidade da atenção prestada aos usuários (2).

O estudo foi realizado por quarenta e uma instituições de ensino superior (IES), lideradas por oito instituições - UFPEL (Universidade Federal de 
Pelotas), UFRGS (Universidade Federal do Rio Grande do Sul), FIOCRUZ (Fundação Oswaldo Cruz), UFMG (Universidade Federal de Minas Gerais), UFBA (Universidade Federal da Bahia), UFRN (Universidade Federal do Rio Grande do Norte), UFS (Universidade Federal do Sergipe) e UFPI (Universidade Federal do Piauí).

Trata-se de um estudo transversal com abordagem quantitativa, cuja amostra foi constituída por equipes de atenção básica indicadas pela gestão municipal para o processo de avaliação externa do Programa e de usuários vinculados a estas equipes e presentes na Unidade Básica de Saúde (UBS) no dia da avaliação. Foram aplicados três instrumentos: o primeiro observou as condições de infraestrutura das UBS; o segundo caracterizou a organização dos serviços e o processo de trabalho das equipes de atenção básica e o terceiro investigou o acesso, a utilização e a qualidade da atenção segundo a percepção dos usuários.

A coleta de dados foi realizada por entrevistadores previamente capacitados, por meio de formulários eletrônicos disponibilizados em tablets. A resolução das inconsistências e as críticas dos dados foram responsabilidade de cada uma das seis instituições que lideraram o processo de avaliação externa do PMAQ.

O desfecho "satisfação dos usuários com o serviço" foi composto pela resposta negativa à pergunta: "se pudesse, mudaria de equipe ou unidade de saúde?", somado à resposta positiva à pergunta: "você recomendaria esta unidade de saúde para um amigo ou familiar?". Como variáveis independentes, foram utilizadas: região geopolítica (Norte, Nordeste, Centro-Oeste, Sudeste e Sul); porte populacional do município, em habitantes (até 10.000; 10.001- 30.000; 30.001-100.000; 100.001-300.000; mais de 300.000); cobertura de estratégia de saúde da família - ESF dos municípios (até 50\%; 50,01-75,0\%; 75,01-99,9\%; 100\%); Índice de Desenvolvimento Humano Municipal (IDH-M) (muito alto-0,862-0,800; alto-0,799-0,700; médio -0,699-0,555; baixo -0,554-0,350); sexo (feminino; masculino); cor da pele auto referida (branca; preta; parda e mestiça; amarela, indígena ou ignorado); escolaridade (nenhuma/analfabeto; fundamental incompleto; fundamental completo/médio incompleto; médio completo/superior incompleto; superior completo/pós-graduação); idade (18 a 44 anos; 45 a 59; 60 a 69; 70 e mais).

Também foram incluídas como variáveis independentes perguntas relacionadas a infraestrutura da UBS, ao processo de trabalho e a autopercepção do cuidado pelo usuário. A infraestrutura foi considerada adequada quando a UBS referiu presença de cinco equipamentos definidos como minimamente necessários para o cuidado de pacientes hipertensos e diabéticos na UBS. Para avaliação do processo de trabalho, foi desenvolvido um escore com nove perguntas, e considerado processo de trabalho adequado a resposta afirmativa a todas às perguntas. Da mesma forma, a variável percepção da qualidade do cuidado clínico foi construída a partir da soma de respostas para nove perguntas, e considerou-se positiva quando todas receberam respostas afirmativas (Quadro 1).

Para análise de dados, inicialmente foi realizada análise descritiva com cálculo das prevalências e respectivos intervalos de confiança (95\%) de cada uma das variáveis de interesse. Para avaliação dos fatores associados foi realizada análise bruta e ajustada. Na análise bruta foram empregados testes de qui-quadrado para heterogeneidade ou tendência linear. A análise ajustada foi realizada com o objetivo de avaliar a associação do desfecho com as variáveis independentes, empregando-se um modelo hierárquico em quatro níveis, controlando os possíveis fatores de confusão. 0 primeiro nível foi composto por variáveis de contexto; no segundo nível entrou a variável infraestrutura da UBS; o terceiro nível foi composto pelas variáveis sociodemográficas e processo de trabalho; e no quarto nível a variável percepção da qualidade do cuidado clínico. $\mathrm{Na}$ análise ajustada foram incluídas inicialmente todas as variáveis do modelo hierárquico. Utilizou-se ajuste backward por níveis hierárquicos, permanecendo no modelo apenas as variáveis com $p \leq 0,20$, através da regressão de Poisson com ajuste robusto da variância. Foram calculadas as razões de prevalência (RP) e respectivos intervalos de 


\section{ARTIGOS}

\section{APS em Revista}

Vol. 1, n. 3, p. 206/221 | Setembro/Dezembro - 2019

ISSN 2596-3317 - DOI 10.14295/aps.v1i3.49

Saez, M. O.; Facchini, L. A.; Tomasi, E.

confiança (IC95\%) e mensurados os valores $p$ do teste de Wald de heterogeneidade e tendência linear. Associações com valor- $p \leq 0,05$ foram consideradas estatisticamente significativas. As análises foram realizadas através do pacote estatístico Stata 12.0.
O estudo foi aprovado pelo Comitê de Ética em Pesquisa da Faculdade de Medicina da Universidade Federal de Pelotas sob o protocolo 21494013.0.0000.5317. Todos os participantes assinaram Termo de Consentimento Livre e Esclarecido.

Quadro 1: Descrição das questões que compuseram as variáveis infraestrutura da UBS, Processo de trabalho e Percepção da qualidade do cuidado clínico.

\begin{tabular}{|c|c|}
\hline Variáveis & Perguntas que compuseram o escore \\
\hline \multirow{5}{*}{ Infraestrutura da UBS } & Há esfigmomanômetro? \\
\hline & Há balança de adulto? \\
\hline & Há régua antropométrica? \\
\hline & Há estetoscópio? \\
\hline & Há glicosímetro? \\
\hline \multirow{8}{*}{ Processo de trabalho } & $\begin{array}{l}\text { A equipe de atenção básica realiza monitoramento e análise dos indicadores e } \\
\text { informações de saúde? }\end{array}$ \\
\hline & A equipe realiza acolhimento à demanda espontânea nesta unidade? \\
\hline & A equipe realiza atendimento de crise hipertensiva? \\
\hline & A equipe realiza atendimento de hiperglicemia? \\
\hline & $\begin{array}{l}\text { O usuário consegue sair da unidade com a consulta marcada nas situações em } \\
\text { que são o caso de atender no mesmo dia? }\end{array}$ \\
\hline & A equipe possui registro do seu território de pessoas com hipertensão? \\
\hline & A equipe possui registro do seu território de pessoas com diabetes? \\
\hline & $\begin{array}{l}\text { A programação da agenda da equipe está de acordo com o risco classificado para } \\
\text { hipertensão? }\end{array}$ \\
\hline
\end{tabular}




\section{APS em Revista}

Vol. 1, n. 3, p. 206/221 | Setembro/Dezembro - 2019

ISSN 2596-3317 - DOI 10.14295/aps.v1i3.49

Saez, M. O.; Facchini, L. A.; Tomasi, E.

\begin{tabular}{|c|c|}
\hline & $\begin{array}{l}\text { A programação da agenda da equipe está de acordo com o risco classificado para } \\
\text { diabetes? }\end{array}$ \\
\hline \multirow{9}{*}{$\begin{array}{l}\text { Percepção da qualidade } \\
\text { do cuidado clínico }\end{array}$} & $\begin{array}{l}\text { Quando é atendido(a) nesta unidade, o(a) senhor(a) acha que a equipe busca } \\
\text { resolver suas necessidades/problemas na própria unidade de saúde? }\end{array}$ \\
\hline & O consultório para o atendimento é um lugar reservado (tem privacidade)? \\
\hline & $\begin{array}{l}\text { Nas consultas, os profissionais da equipe fazem o exame físico, examinam o } \\
\text { seu corpo, sua garganta, a sua barriga? }\end{array}$ \\
\hline & $\begin{array}{l}\text { Para ajudar na sua recuperação, os profissionais orientam o senhor(a) em } \\
\text { relação a necessidade de repouso, alimentação adequada e como tomar os } \\
\text { remédios? }\end{array}$ \\
\hline & $\begin{array}{l}\text { Os profissionais de saúde orientam o senhor(a) sobre o que fazer quando os seus } \\
\text { sintomas estão piorando? }\end{array}$ \\
\hline & $\begin{array}{l}\text { Além da sua queixa os profissionais de saúde perguntam sobre outras questões } \\
\text { da sua vida (exemplos: alimentação, lazer, exercício físico, problemas com } \\
\text { álcool, drogas, violência)? }\end{array}$ \\
\hline & $\begin{array}{l}\text { O(a) senhor(a) se sente à vontade para falar com a equipe sobre suas } \\
\text { preocupações? }\end{array}$ \\
\hline & $\begin{array}{l}\text { O(a) senhor(a) se sente respeitado(a) pelos profissionais em relação aos seus } \\
\text { hábitos culturais, costumes, religião? }\end{array}$ \\
\hline & $\begin{array}{l}\text { Na opinião do(a) senhor(a), durante as consultas, os profissionais desta equipe } \\
\text { sugerem soluções possíveis de serem realizadas? }\end{array}$ \\
\hline
\end{tabular}

Fonte: elaboração dos autores

\section{RESULTADOS}

Com dados do segundo ciclo do PMAQ, foram avaliadas 29.000 equipes de saúde e entrevistados 113.272 usuários, dos quais 42.994 apresentavam diagnóstico de hipertensão e 15.557 de diabetes. Dos 47.927 usuários hipertensos e/ou diabéticos identificados, a maioria residia na região Sudeste $(40,4 \%)$ e em municípios com população entre 10.001 a 30.000 (26,0\%). Aproximadamente $60,0 \%$ vivia em municípios com elevada cobertura de ESF (>75\%) e com IDH-M alto e muito alto (Tabela 1). Com relação às características sociodemográficas, a maioria dos entrevistados era do sexo feminino $(73,5 \%)$, de cor da pele parda ou mestiça $(42,6 \%)$, com idade entre 45 e 59 anos (56,3\%), e com ensino fundamental incompleto (42,3\%). A percepção da qualidade do cuidado foi considerada positiva por 20,1\% dos usuários hipertensos e/ou diabéticos (Tabela 1) e os itens que mais impactaram negativamente no escore foram: "nas consultas, os profissionais fazem o exame físico, examinam o seu corpo, sua garganta e barriga?" (46,6\%) e "os profissionais de saúde perguntam sobre outras questões de sua vida (alimentação, lazer, exercício físico)?" (51,5\%) (Figura 3). 
Cerca de três quartos das UBS apresentavam estrutura adequada para o cuidado dos usuários hipertensos e/ou diabéticos (73,3\%). Quando observados individualmente os itens que compuseram esta variável, glicosímetro, estetoscópio, régua antropométrica e esfigmomanômetro superaram $90 \%$ de disponibilidade, enquanto balança de adulto foi verificada em apenas $85,9 \%$ dos serviços (Figura 1).

O processo de trabalho foi considerado adequado em 52,9\% das equipes. Neste escore, os itens menos frequentes foram a programação da agenda da equipe de acordo com o risco para diabetes $(66,4 \%)$ e hipertensão $(66,5 \%)$ (Figura 2$)$.

A prevalência de satisfação dos usuários hipertensos e/ou diabéticos com o serviço foi de 80,3\% (IC95\% 79,9-80,6). Observou-se que a satisfação foi maior nas regiões Sul $(85,1 \%$; IC95\% $84,2-85,9)$ e Sudeste (81,8\%; IC95\% 81,2-82,3), em municípios menores (82,1\%; IC95\% 81,1\%-83,1), com maior IDH-M (81,9\%; IC95\% 80,9-82,9) e municípios com maiores coberturas de ESF (81,7\%; IC95\% 81,1-82,3).

Usuários do sexo masculino (84,3\%; IC95\% 83,9$84,7)$, de cor da pele branca (83,5\%; IC95\% 82,9$84,0)$, com maior idade (85,3\%; IC95\% 84,5-86,1) e maior escolaridade (85,6\%; IC95\% 83,5-87,5) estavam mais satisfeitos com o serviço. A satisfação também foi maior em unidades com infraestrutura adequada (80,9\%; IC95\% 80,5-81,3), quando o processo de trabalho das equipes foi adequado (82,8\%; IC95\% 82,3-83,3) e também quando a percepção da qualidade do cuidado clínico era positiva (94,3\%; IC95\% 93,8-94,8) (Tabela 1).

Após ajustes, a satisfação dos usuários hipertensos e/ou diabéticos na rede básica foi 10,0\% maior na região Sul (IC95\% 1,07-1,13) e 14,0\% maior nos municípios com maior IDH-M (IC95\% 1,10-1,18). Usuários homens, de cor da pele branca, com mais idade e com maior escolaridade apresentaram maior probabilidade de estarem satisfeitos, sugerindo um efeito dose-resposta para idade e escolaridade. A inadequação da infraestrutura e do processo de trabalho reduziram, ainda que pouco, a satisfação dos usuários. Contudo, observou-se que a satisfação foi fortemente afetada pela percepção negativa dos usuários sobre o cuidado clínico recebido no serviço, tendo reduzido em $17,0 \%$ (IC95\% 0,82-0,84) a satisfação, independente de todas as características - de contexto, de estrutura e processo de trabalho e sociodemográficas - que poderiam confundir esta associação.

Não foi identificada associação entre o desfecho e as variáveis porte populacional e cobertura de ESF dos municípios.

Examinado a associação entre a satisfação e cada uma das variáveis que compuseram o escore de percepção da qualidade do cuidado clínico, duas delas se destacaram: a prevalência de satisfação foi cerca de 50,0\% maior entre usuários que perceberam positivamente a presença de consultório com privacidade - $81,8 \%$ versus $52,2 \%$ e $82,4 \%$ versus $59,6 \%$ para os que referiram respeito aos hábitos culturais e religiosos.

\section{Discussão}

O estudo avaliou a satisfação dos usuários com hipertensão e/ou diabetes com o cuidado recebido na atenção básica no ciclo II do PMAQ. Apesar da prevalência de satisfação ter sido alta $(80,3 \%)$, o que mais influenciou foi a percepção da qualidade do cuidado acerca da prática clínica.

Protasio et al (2017), encontraram prevalência semelhante $(77,0 \%)$ ao avaliar a satisfação de todos os usuários no ciclo I do PMAQ. A satisfação é um importante indicador da qualidade do cuidado ofertado, tanto quanto ao acesso, como ao processo de trabalho, impactando diretamente na adesão e efetividade do tratamento dos usuários $(5,11)$. Uma vez que o cuidado aos usuários com hipertensão e/ou diabetes é uma das ações programáticas preconizadas pelo Ministério da Saúde e sua qualidade foi aferida por ocasião da avaliação externa do PMAQ, seria plausível supor que a satisfação destas pessoas fosse maior do que a de usuários de demanda espontânea. Contudo, a prevalência de satisfação é alta, independente do grupo populacional avaliado 9,15. Diferentes estudos ponderam que o elevado sentimento de 
satisfação pode ser causado pela baixa exigência individual dos usuários. Além disso, um sentimento de gratidão poderia impedir que os usuários avaliassem mais criticamente os serviços recebidos devido ao receio de fragilizar o vínculo com o profissional de saúde e limitar o acesso aos cuidados recebidos (16-18).

Em que pese a existência de diferentes medidas da satisfação na literatura - escala de faces, perguntas abertas- a forma como esse estudo aferiu a satisfação - por meio de respostas combinadas a duas perguntas - parece não ter acrescentado nenhum tipo de viés aos resultados, pois houve bastante similaridade com os resultados encontrados em outros estudos $(9,19)$.

A variabilidade na opinião dos usuários, embora pequena, foi significativa e coincide com as inequidades expressas na infraestrutura e na qualidade dos serviços de APS no Brasil. A maior satisfação ocorreu nas regiões Sul e Sudeste, as mais ricas do país e em municípios situados em estratos de IDH mais elevados. Esta relação não foi observada com o porte populacional e a cobertura de ESF do município, após o ajuste simultâneo no mesmo nível hierárquico. Região geopolítica e IDH sintetizam as marcantes diferenças nas condições de infraestrutura urbana e social e na maior cobertura de serviços educacionais e de saúde, características contextuais que também estiveram associadas à maior satisfação de usuários em outros estudos (7). $O$ achado reforça a necessidade de manutenção de políticas públicas que monitorem e avaliem os esforços de combate às inequidades sociais em saúde, a exemplo do PMAQ 20.

Os achados deste estudo também apontaram maior satisfação entre os homens, indivíduos de cor da pele branca e entre aqueles com maior escolaridade, resultado semelhante ao encontrado em outras pesquisas (20-23). Quanto ao sexo, diferentes estudos reforçam que as mulheres utilizam com maior frequência os serviços de saúde, e justificam o achado pelas diferentes demandas específicas do sexo (menarca, gestação, menopausa) e também por que as mulheres apresentam maior interesse com relação à sua saúde $(22,24)$. O uso mais frequente do serviço de saúde pode fazer com que as mulheres percebam melhor as fragilidades do serviço, o que pode afetar sua percepção de satisfação (7). Uma vez que já está descrita a associação entre cor da pele e escolaridade em nosso país, também encontrada nos dados do presente estudo, a maior satisfação registrada entre usuários de cor da pele branca pode sugerir um efeito sinérgico com a escolaridade, apesar do ajuste simultâneo no modelo multivariado. Gouveia et al (2009) pontuam que a maior escolaridade altera positivamente a capacidade de entendimento das informações de saúde, o acesso, a utilização dos serviços de saúde e a adesão ao tratamento, afetando diretamente a percepção de satisfação com os serviços.

Romero et al (2016) ao avaliar a satisfação de usuários da atenção primária a saúde na Espanha, também identificou um aumento gradual da satisfação com o incremento da idade, e relata que este resultado independe da cultura, do país e do tipo de organização do serviço, podendo ser justificado pela maior necessidade e uso do serviço e pelo sentimento de gratidão acerca do cuidado recebido.

Donabedian (2005) propôs a tríade estrutura, processo de trabalho e resultados para avaliar a qualidade dos serviços de saúde, sendo a infraestrutura e o processo de trabalho adequados indispensáveis para alcançar resultados satisfatórios na situação de saúde dos indivíduos e em seus níveis de satisfação $(3,25)$. Estudos realizados no Brasil e no exterior para avaliação da satisfação dos usuários com o serviço ofertado na atenção básica também evidenciaram relação direta entre melhoria da infraestrutura do estabelecimento e do processo de trabalho das equipes e maior a satisfação manifestada pelos usuários (19,26-27). Nesse estudo, optou-se por trabalhar com medidas sintéticas para investigar a adequação da estrutura dos serviços e do processo de trabalho das equipes, construídas com variáveis disponíveis no instrumento do PMAQ. Esta abordagem permitiu a análise dos elementos da tríade proposta por Donabedian, a partir da qual constatou-se que usuários atendidos em unidades de saúde com infraestrutura adequada e por 
equipes com processo de trabalho adequado estavam mais satisfeitos do que os demais.

A percepção do usuário sobre a qualidade do cuidado clínico recebido foi o fator mais fortemente associado com a satisfação, ajustando-se para as demais variáveis independentes. A satisfação foi $22 \%$ maior entre quem teve uma percepção positiva deste cuidado. Este resultado sugere que usuários atendidos por profissionais que se dediquem mais ao exame físico, que demonstrem maior interesse com as demandas individuais e familiares, que forneçam mais orientações e que se preocupem com o cuidado continuado conseguem estabelecer melhor vínculo com as equipes, o que afeta positivamente a sua satisfação (28-31). Este vínculo se reveste de maior importância se consideramos que a satisfação foi aferida por meio de perguntas sobre a permanência na equipe e sua indicação para outras pessoas. Corroborando com nosso achado, um estudo de revisão sistemática acerca dos determinantes da satisfação dos pacientes, identificou que a melhor qualidade do atendimento interpessoal foi o indicador com maior impacto na satisfação dos indivíduos com o serviço (19)

Uma das limitações do estudo é a possibilidade de viés de seleção da amostra, pois o PMAQ é um programa por adesão, o que pode ter levado a maior participação de equipes com melhor processo de trabalho e qualidade do cuidado. Esta característica foi marcante no ciclo 1 , pois nos ciclos 2 e 3 o PMAQ tornou-se progressivamente universal, alcançando praticamente a totalidade das equipes da ESF em 2018. Além disso, o grande tamanho da amostra facilita estatisticamente refutar a hipótese nula, indicando associação entre todas as variáveis investigadas, mesmo após o controle de fatores de confusão. Para esta limitação, optou-se por avaliar os resultados a partir das razões de prevalência e seus respectivos intervalos de confiança.

Os resultados deste estudo reforçam que o principal determinante da satisfação é a percepção do usuário sobre o cuidado recebido no serviço, fortalecendo a relevância do investimento em qualificação e educação permanente, maior valorização e menor carga de trabalho dos profissionais da atenção básica, promovendo condições para a oferta de serviços com enfoque completo, complexo e humanizado com proposições terapêuticas construídas juntamente com o usuário e adequadas ao seu contexto.

A avaliação de satisfação dos usuários é valiosa para o conhecimento de gestores, profissionais de saúde e população sobre a capacidade de resposta dos serviços às demandas e necessidades de saúde $(6,32)$.

\section{REFERÊNCIAS BIBLIOGRÁFICAS}

1. BRASIL. Ministério da Saúde. Secretaria de Atenção à Saúde. Departamento de Atenção Básica. Política Nacional de Atenção Básica / Ministério da Saúde. Secretaria de Atenção à Saúde. Departamento. Brasília; 2017. 399-404 p.

2. BRASIL. Ministério da Saúde. Programa Nacional de Melhoria do Acesso e da Qualidade da Atenção Básica: manual instrutivo. 2011;44p.

3. Donabedian A. Evaluating the quality of medical care. Milbank Quartely. 2005;83(4):691-729.

4. Silva CSO, Fonseca ADG, Souza LPS, Siqueira LG, Belasco AGS, Barbosa DA. Integralidade e Atenção Primária à Saúde: avaliação sob a ótica dos usuários. Cien Saude Colet. 2014;19(11):4407-15.

5.Paes NA, Silva CS, Figueiredo TMRM, Cardoso MAA, Oliveira JL. Satisfação dos usuários hipertensos com os serviços da rede de atenção primária no Brasil: um estudo de validação. Rev Panam Salud Publica/Pan Am J Public Heal. 2014;36(2):87-93.

6.Al-Assaf AF, Sheikh M. (editors). Quality improvement in primary health care: a practical guide. Cairo, WHO Regional Publications, Eastern Mediterranean Series ; 26), 2004. ISBN 92-9021296-9

7. Romero SP, Cánovas JJG, Martínez DS, Hidalgo PP, Piqueres OM. Características sociodemográficas y variabilidad geográfica relacionada com la satisfacción del paciente em Atención Primaria. Rev Calid Asist. 2016;31(5):300-308.

8. Raivio R, Jaaskelainen J, Marttila DH, Martila KJ. 


\section{APS em Revista}

Vol. 1, n. 3, p. 206/221 | Setembro/Dezembro - 2019

ISSN 2596-3317 - DOI 10.14295/aps.v1i3.49

Saez, M. O.; Facchini, L. A.; Tomasi, E.

Decreasing trends in patient satisfaction, acessibility and continuity of care in Finnish primary helath care - a 14 year follw up questionnaire study. BMC Family Practice 2014, 15:98.

9. Protasio APL, Gomes LB, Machado LS, Valença AMG. Factors associated with user satisfaction regarding treatment offered in Brazilian primary health care. Cad Saude Publica. 2017;33(2):1-15.

10. Paula WKAS, Samico IC, Caminha MFC, Filho MB, Silva SL. Avaliação ABS sob optica dos usuarios Brasil. 2016;50(2):335-45.

11. Redes de atenção à saúde : práticas, experiências e propostas na gestão da Saúde Coletiva [recurso eletrônico] / Organizadores: Alexandre Fávero Bulgarelli [et al.] - 1.ed. - Porto Alegre : Rede UNIDA, 2016.

12. Macinko J, Guanais FC, Mullachery P, Jimenez G. Gaps in primary care and health system performance in six Latin American and Caribbean Countries. Health Affairs, 2016; 35(8):1513-1521.

13. Forti AC, Pereira AM S, Amodeo C, Filho FF, Praxedes JN, Mota MA, et al. Cadernos de Atenção Básica. Vol. 7, Ministério da Saúde. 2001. 1-96 p.

14. Savassi LCM. Qualidade em serviços públicos: os desafios da atenção primária. Rev Bras Med Família e Comunidade. 2012;7(23):69-74.

15. Vieira AST, Miranda MSL, Emmi DT, Pinheiro HHC, Barroso RFF, Araújo MVA. Percepção dos usuários de serviços de saúde da atenção b'sica no estado do Pará. Rev. Bras. Pesq. Saúde. 2016;18(3):58-64.

16. Bernhart $\mathrm{MH}$, Wiadnyana IGP, Wihardjo $\mathrm{H}$, Pohan I. Patient satisfaction in developing countries. Soc Sci Med. 1999; 48(8):989-996.

17. Vaitsman J, Andrade GRB, Farias LO, Médici ME \& Nascimento LR 2003. Projeto Gestão de Qualidade e Satisfação dos Usuários em Organizações Públicas de Saúde. Um estudo piloto no Instituto de Pesquisa Evandro Chagas - IPEC. Resumo do relatório final a pre $s$ en $t$ ado à Fundação de Amparo à Pesquisa do Rio de Ja $n$ ei ro - FAPERJ. ENSP/ Fiocruz.

18. Villa TCS, Andrade RLP, Arakawa T, Magnabosco GT, Beraldo AA, Monroe AA, Ponce MAS, Brunello MEF, Scatena LM, Palha PF, Ruffino-Netto A.
Satisfação do usuário com os serviços de atenção à tuberculose em Ribeirão Preto, 2008. Cad. Saúde Colet. 2012;20(2): 234-43.

19. Batbaatar E, Dorjdagva J, Luvsannyam A, Savino $M$, Amenta P. Determinants of patient satisfaction: a systematic review.Perpectives in Public Health. 2017;137(2):89-101.

20. Gouveia GC, Souza WV, Luna CF, Souza-Junior PRB, Scwarcwald CL. Satisfação dos usuários do sistema de saúde brasileiro: fatores associados e diferenças regionais. Rev. Bras. Epidemiol. 2009;12(3): 281-96.

21. Weiss GL 1988. Patient satisfaction with primary medical care: evaluation of sociodemographic and predispositional factor. Medical Care 1988;26(4): 383-392.

22. Travassos C, Oliveira EXG, Viacava F. Desigualdades geográficas e sociais no acesso aos serviços de saúde no Brasil: 1998 e 2003. Ciên Saúde Coletiva 2006; 11(4): 975- 86.

23. Nunes BP, Thumé E, Tomasi E, Duro SMS, Facchini LA. Desigualdades socioeconômicas no acesso e qualidade da atenção nos serviços de saúde. Revista de Saúde Pública. 2014; 48(6): 968976.

24. Pinheiro RS, Viacava F, Travassos C, Brito AS. Gênero, morbidade, acesso e utilização de serviços de saúde no Brasil. Ciência \& Saúde Coletiva. 2002; 7(4):687-707.

25. Neves RG, Duro SMS, Muniz J, Castro TRP, Facchini LA, Tomasi E. Estrutura das unidades básicas de saúde para atenção às pessoas com diabetes: Ciclos I e II do Programa Nacional de Melhoria do Acesso e da Qualidade. Cad. Saúde Pública 2018; 34(4):e00072317

26. Esperidião M, Trad LAB. Avaliação de satisfação de usuários. Ciência \& Saúde Coletiva. 2005; 10(Sup):303-312.

27. Jorge MSB, Guimarães JMX, Vieira LB, Paiva FDS, Silva, DR, Pinto AGA. Avaliação da qualidade do Programa Saúde da Família no Ceará: a satisfação dos usuários. Rev. Baiana Saúde Pública. 2007;31(2):256-266.

28. Moimaz SAS, Marques JAM, Saliba O, Garbin CAS, Zina LG, Saliba NA. Satisfação e percepção do 
Vol. 1, n. 3, p. 206/221 | Setembro/Dezembro - 2019

ISSN 2596-3317 - DOI 10.14295/aps.v1i3.49

Saez, M. O.; Facchini, L. A.; Tomasi, E.

usuário o SUS sobre o serviço público de saúde. Physis Revista de Saúde Coletiva. 2010; 20(4): 14191440.

29. Abreu DMX, Araújo LHL, Reis CMR, Lima AMLD, Santos AF, Jorge AO, Sobrinho DF, Machado ATGM. Service users perception about healthcare provided by teams participating in the National Primary Care Access and Quality Improvement in Brazil. Epidemiol. Serv. Saude. 2018; 27(3):32017111

30. Vinogradova $Y$, Coupland $C$, Brindle $P$, HippisleyCox J. Discontinuation and restarting in patients on statin treatment: Prospective open cohort study using a primary care database. BMJ. 2018; 28(353):i3305.

31. Fausto MCR, Bousquat A, Lima JG, Giovanella L, Almeida PF, Mendinça MHM, Seidi $H$, Silva ATC. Evalution of Brazilian Primary Health Care From the Perspective od Users: Acessible, Continuous ans Acceptable? J Ambulatory Care Manage, 2017; 40(2): 560-570.

32. Facchini LA, Tomasi E, Dilélio AS. Qualidade da Atenção Primária à Saúde no Brasil: avanços, desafios e perspectivas. Saúde em Debate. 2018;42(spe1):208-223. doi:10.1590/010311042018 s114 


\section{APS em Revista}

Vol. 1, n. 3, p. 206/221 | Setembro/Dezembro - 2019

ISSN 2596-3317 - DOI 10.14295/aps.v1i3.49

Saez, M. O.; Facchini, L. A.; Tomasi, E.

\section{APÊNDICE}

Figura 1: Distribuição dos insumos e materiais que compuseram a variável infraestrutura da UBS, PMAQ ciclo II, 2014.

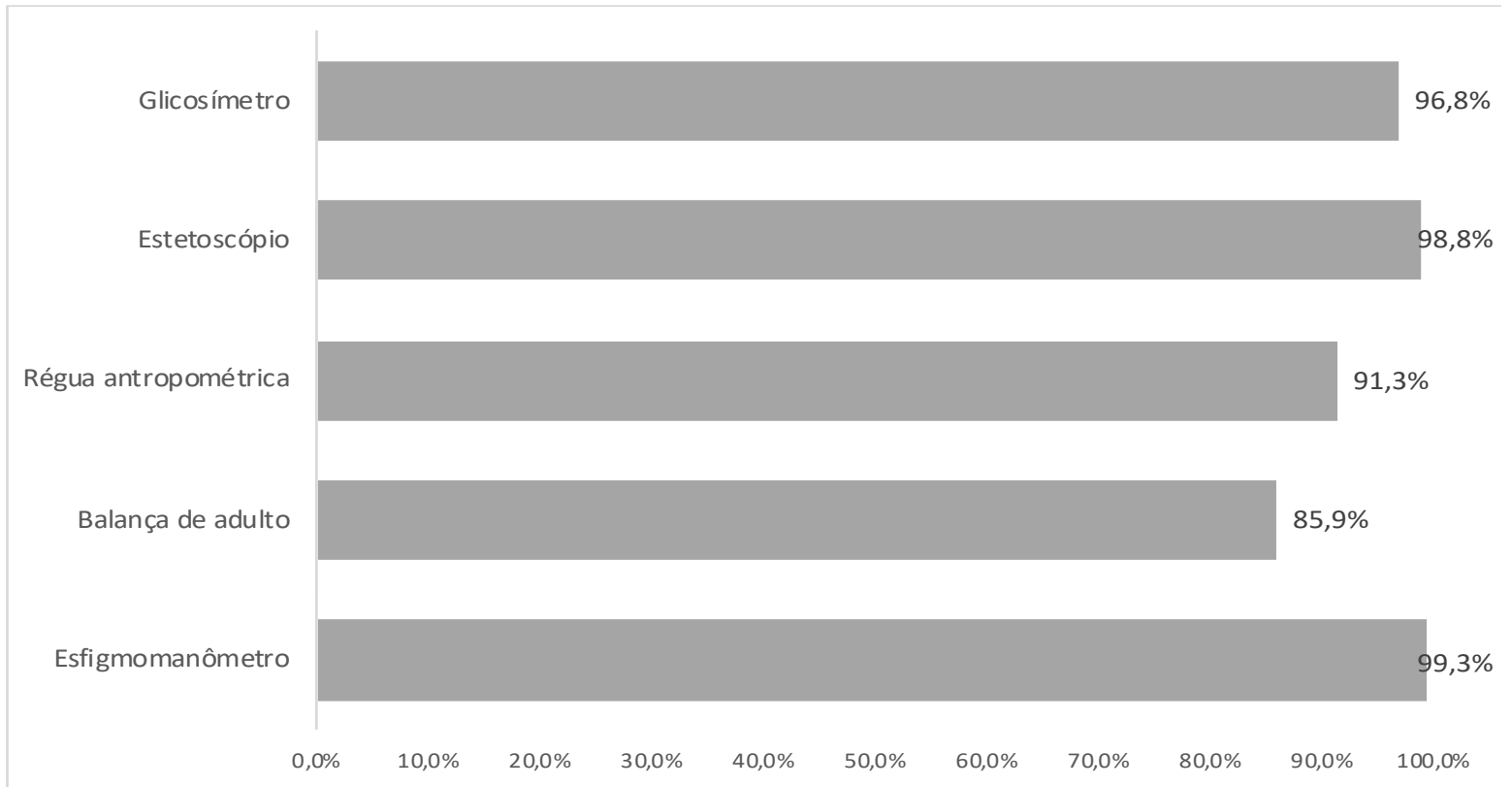

Figura 2: Distribuição das perguntas que compuseram a variável processo de trabalho, PMAQ ciclo II, 2014.

A programação da agenda da equipe está de acordo com o risco classificado para diabetes?

A programação da agenda da equipe está de acordo com o risco classificado para hipertensão?

A equipe possui registro do seu território de pessoas com diabetes?

A equipe possui registro do seu território de pessoas com hipertensão?

O usuário consegue sair da unidade com a consulta marcada nas situações em que são o caso de atender no mesmo dia?

A equipe realiza aten dimento de hiperglicemia?

A equipe realiza aten dimento de crise hiperten siva?

A equipe realiza acolhimento à demanda espontânea nesta unidade?

A equipe de atenção básica realiza monitoramento e análise dos indicadores e informações de saúde?
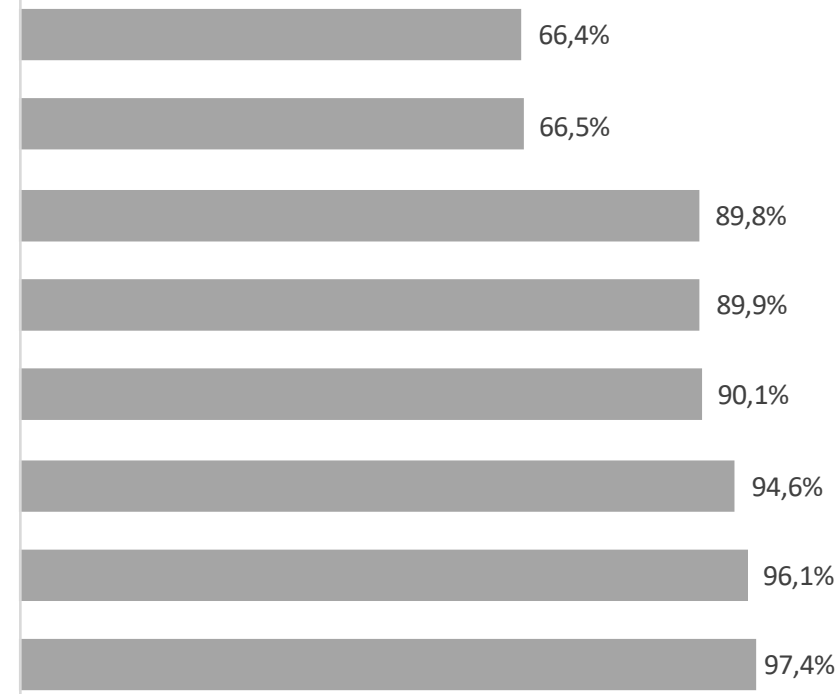

$97,4 \%$

$89,1 \%$

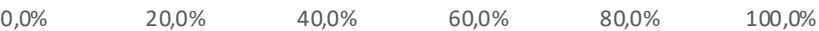


APS em Revista

Vol. 1, n. 3, p. 206/221 | Setembro/Dezembro - 2019

ISSN 2596-3317 - DOI 10.14295/aps.v1i3.49

Saez, M. O.; Facchini, L. A.; Tomasi, E.

Figura 3: Distribuição das perguntas que compuseram a variável percepção da qualidade do cuidado clínico, PMAQ ciclo II, 2014.

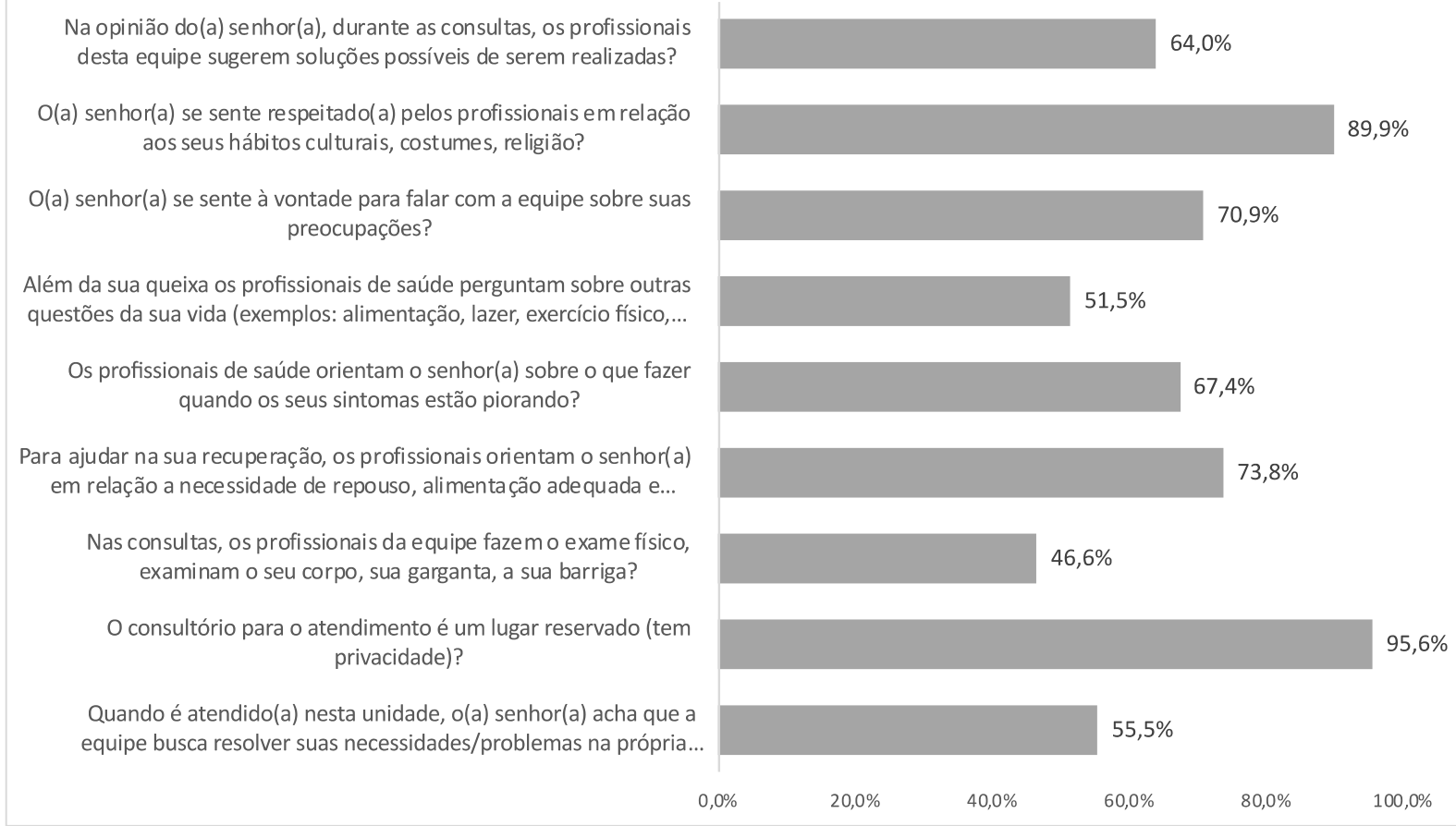

Tabela 1: Distribuição da amostra e prevalência de satisfação entre usuários portadores de hipertensão e diabetes da Atenção Básica - PMAQ, Brasil, 2014 (n=47.927).

\begin{tabular}{lcccc}
\hline \multicolumn{1}{c}{ Variáveis } & \multicolumn{2}{c}{ Amostra } & \multicolumn{2}{c}{ Prevalência de Satisfação } \\
& $\mathbf{n}$ & $\%$ & $\mathbf{n}$ & \% (IC95\%) \\
\hline Região (47.927) & & & & \\
Norte & 2.615 & 5,5 & 1.941 & $74,2(72,5-75,9)$ \\
$\quad$ Nordeste & 14.099 & 29,4 & 10.843 & $76,9(76,2-77,6)$ \\
Sudeste & 19.341 & 40,4 & 15.814 & $81,8(81,2-82,3)$ \\
$\quad$ Sul & 7.573 & 15,8 & 6.443 & $85,1(84,2-85,9)$ \\
$\quad$ Centro-Oeste & 4.299 & 8,9 & 3.450 & $80,3(79,0-81,5)$ \\
Porte populacional (habitantes) (47.822) & & & & \\
$\quad$ S10.000 & 6.075 & 12,7 & 4.935 & $82,1(81,1-83,1)$ \\
10.001 a 30.000 & 12.420 & 26,0 & 10.019 & $82,1(81,4-82,8)$ \\
30.001 a 100.000 & 10.537 & 22,0 & 8.438 & $80,8(80,0-81,5)$ \\
\hline
\end{tabular}


Vol. 1, n. 3, p. 206/221 | Setembro/Dezembro - 2019

ISSN 2596-3317 - DOI 10.14295/aps.v1i3.49

Saez, M. O.; Facchini, L. A.; Tomasi, E.

\begin{tabular}{ccccc}
\hline 100.001 a 300.000 & 6.764 & 14,1 & 5.349 & $79,7(78,7-80,6)$ \\
$>$ > 300.000 & 12.026 & 25,2 & 9.249 & $77,7(76,9-78,4)$ \\
Cobertura de ESF dos municípios (47.828) & & & & \\
Até 50\% & 10.613 & 22,2 & 8.376 & $79,4(78,6-80,2)$ \\
50,01 a 75,00\% & 9.206 & 19,3 & 7.142 & $78,6(77,7-79,4)$ \\
75,01 a 99,99\% & 9.958 & 20,8 & 7.950 & $80,6(79,8-81,4)$ \\
100\% & 18.051 & 37,7 & 14.527 & $81,7(81,1-82,3)$
\end{tabular}

IDH-M (47.827)

Baixo
Médio
Alto

Muito alto

Infraestrutura da ESF (47.828)

$$
\begin{aligned}
& \text { Adequada } \\
& \text { Inadequada }
\end{aligned}
$$

Feminino

Cor da pele auto referida (47.013)

Branca
Preta
Parda e Mestiça
Amarela/Indígena

Escolaridade (47.836)

Nenhuma/Analfabeto
Fundamental incompleto
Fundamental completo/ Médio
incompleto

Médio completo/ Superior 6.657

incompleto$$
1.606
$$$$
19.079
$$$$
21.272
$$$$
5.870
$$

24.619

21.960

12.682

26,5

73,5

35.245

11.899

20.229

7.825

18.181

38,6

14,7

42,6

4,5

4.786

76,2 (74,1-78,3)

17.200

$78,8(78,2-79,4)$

14.836

$81,6(81,1-82,1)$

1.173

$81,9(80,9-82,9)$

12,3

73,3

28.080

$80,9(80,5-81,3)$

9.915

$78,7(78,0-79,4)$

8.687

$82,8(82,3-83,3)$

34.635

$78,2(77,6-78,7)$

47,1

27.797

$84,3(83,9-84,7)$

10.694

$78,9(78,2-79,6)$

20.017

1.902

24,9

9.525

$80,0(79,3-80,7)$

42,3

16.330

$80,7(80,2-81,2)$

16,3

6.208

$79,3(78,4-80,2)$

14,0

5.313

$79,8(78,8-80,8)$ 


\section{APS em Revista}

Vol. 1, n. 3, p. 206/221 | Setembro/Dezembro - 2019

ISSN 2596-3317 - DOI 10.14295/aps.v1i3.49

Saez, M. O.; Facchini, L. A.; Tomasi, E.

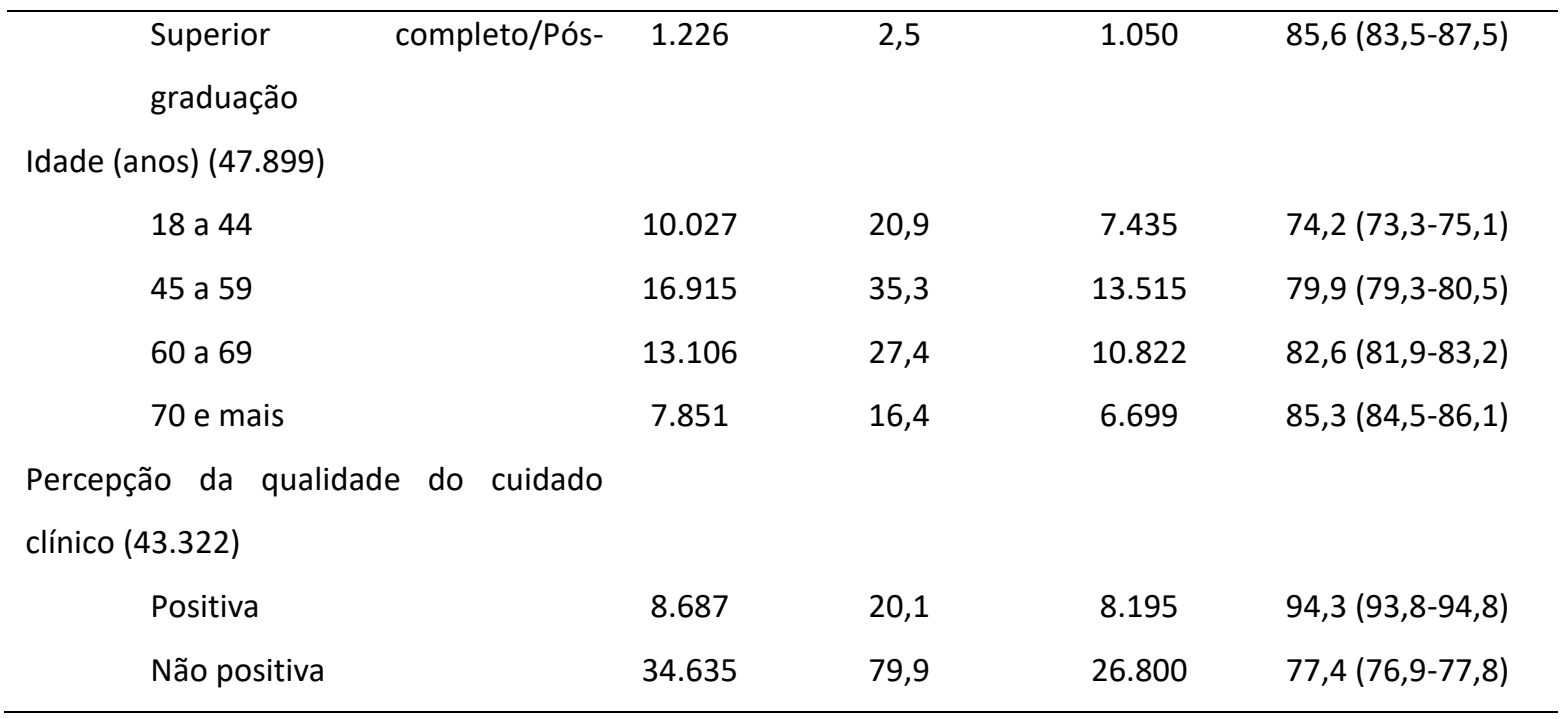

Tabela 2: Prevalência de satisfação, análise bruta e ajustada conforme as variáveis associadas ao desfecho entre usuários portadores de hipertensão e diabetes da Atenção Básica - PMAQ, Brasil, 2014 (n=47.927).

\begin{tabular}{|c|c|c|c|c|c|c|}
\hline \multirow{2}{*}{$\begin{array}{c}\text { Níveis } \\
\text { Hierárquicos }\end{array}$} & \multirow[t]{2}{*}{ Variáveis } & \multirow{2}{*}{$\begin{array}{c}\text { Satisfação } \\
\%\end{array}$} & \multicolumn{2}{|c|}{ Análise Bruta } & \multicolumn{2}{|c|}{ Análise Ajustada } \\
\hline & & & RP & IC95\% & $\mathbf{R P}$ & IC95\% \\
\hline \multirow[t]{22}{*}{ 10 Nível } & Região $\#$ & & & & & \\
\hline & Norte & 74,2 & 1 & & 1 & \\
\hline & Nordeste & 76,9 & 1,04 & $1,01-1,06$ & 1,02 & $1,00-1,05$ \\
\hline & Sudeste & 81,8 & 1,10 & $1,08-1,13$ & 1,08 & $1,05-1,11$ \\
\hline & Sul & 85,1 & 1,15 & $1,12-1,17$ & 1,10 & $1,07-1,13$ \\
\hline & Centro-Oeste & 80,3 & 1,08 & $1,05-1,11$ & 1,05 & $1,03-1,08$ \\
\hline & Porte populacional (habitantes) & & & & & \\
\hline & $<10.000$ & 82,1 & 1 & & 1 & \\
\hline & 10.001 a 30.000 & 82,1 & 1,00 & $0,98-1,01$ & 1,02 & $1,00-1,03$ \\
\hline & 30.001 a 100.000 & 80,8 & 0,98 & $0,97-1,00$ & 1,00 & $0,98-1,02$ \\
\hline & 100.001 a 300.000 & 79,7 & 0,97 & $0,95-0,99$ & 0,97 & $0,95-0,99$ \\
\hline & $>300.000$ & 77,7 & 0,95 & $0,93-0,96$ & 0,91 & $0,89-0,93$ \\
\hline & Cobertura de ESF dos municípios $^{\#}$ & & & & & \\
\hline & Até $50 \%$ & 79,4 & 1 & & 1 & \\
\hline & 50,01 a $75,00 \%$ & 78,6 & 0,99 & $0,98-1,00$ & 0,99 & $0,97-1,00$ \\
\hline & 75,01 a $99,99 \%$ & 80,6 & 1,02 & $1,00-1,03$ & 1,01 & $1,00-1,03$ \\
\hline & $100 \%$ & 81,7 & 1,03 & $1,02-1,04$ & 1,03 & $1,01-1,05$ \\
\hline & $\mathrm{IDH}_{-} \mathrm{M}^{\#}$ & & & & & \\
\hline & Baixo & 76,2 & 1 & & 1 & \\
\hline & Médio & 78,8 & 1,03 & $1,00-1,06$ & 1,03 & $1,00-1,06$ \\
\hline & Alto & 81,6 & 1,07 & $1,04-1,10$ & 1,08 & $1,05-1,12$ \\
\hline & Muito alto & 81,9 & 1,07 & $1,04-1,11$ & 1,14 & $1,10-1,18$ \\
\hline \multirow[t]{2}{*}{ 2o Nível } & Infraestrutura da ESF & & & & & \\
\hline & Adequada & 80,9 & 1 & & 1 & \\
\hline
\end{tabular}


Vol. 1, n. 3, p. 206/221 | Setembro/Dezembro - 2019

ISSN 2596-3317 - DOI 10.14295/aps.v1i3.49

Saez, M. O.; Facchini, L. A.; Tomasi, E.

\begin{tabular}{|c|c|c|c|c|c|c|}
\hline & Inadequada & 78,7 & 0,97 & $0,96-0,98$ & 0,97 & $0,96-0,98$ \\
\hline \multirow[t]{23}{*}{ 3o Nível } & Processo de trabalho"\# & & & & & \\
\hline & Adequado & 20,1 & 1 & & 1 & \\
\hline & Inadequado & 79,9 & 0,94 & $0,94-0,95$ & 0,95 & $0,94-0,96$ \\
\hline & Sexo"\# & & & 0,000 & & \\
\hline & Masculino & 84,3 & 1 & & 1 & \\
\hline & Feminino & 78,9 & 0,94 & $0,93-0,94$ & 0,96 & $0,95-0,97$ \\
\hline & Cor da pele auto referida"\# & & & & & \\
\hline & Branca & 83,5 & 1 & & 1 & \\
\hline & Preta & 77,7 & 0,93 & $0,92-0,94$ & 0,96 & $0,95-0,98$ \\
\hline & Parda e Mestiça & 78,6 & 0,94 & $0,93-0,95$ & 0,98 & $0,97-0,99$ \\
\hline & Amarela/Indígena & 77,9 & 0,93 & $0,91-0,96$ & 0,96 & $0,94-0,99$ \\
\hline & Escolaridade $^{\#}$ & & & & & \\
\hline & Nenhuma/Analfabeto & 80,0 & 1 & & 1 & \\
\hline & Fundamental incompleto & 80,7 & 1,01 & $0,99-1,02$ & 1,02 & $1,01-1,03$ \\
\hline & $\begin{array}{l}\text { Fundamental completo/ } \\
\text { Médio incompleto }\end{array}$ & 79,3 & 0,99 & $0,98-1,01$ & 1,02 & $1,00-1,04$ \\
\hline & Médio completo/ & 79,8 & 1,00 & $0,98-1,01$ & 1,04 & $1,03-1,06$ \\
\hline & Superior incompleto & & & & & \\
\hline & Superior completo/PG & 85,6 & 1,07 & $1,04-1,10$ & 1,09 & $1,06-1,12$ \\
\hline & Idade (anos) ${ }^{\#}$ & & & & & \\
\hline & 18 a 44 & 74,2 & 1 & & 1 & \\
\hline & 45 a 59 & 79,9 & 1,08 & $1,06-1,09$ & 1,08 & $1,06-1,09$ \\
\hline & 60 a 69 & 82,6 & 1,11 & $1,10-1,13$ & 1,11 & $1,09-1,12$ \\
\hline & 70 e mais & 85,3 & 1,15 & $1,13-1,17$ & 1,14 & $1,12-1,16$ \\
\hline \multirow[t]{3}{*}{ 40 Nível } & $\begin{array}{l}\text { Percepção da qualidade do } \\
\text { cuidado clínico \#\# }\end{array}$ & & & & & \\
\hline & Positiva & 94,3 & 1 & & 1 & \\
\hline & Não positiva & 77,4 & 0,82 & $0,81-0,83$ & 0,83 & $0,82-0,84$ \\
\hline
\end{tabular}

${ }^{\#} \mathrm{p}$-valores de tendência linear $<0,001{ }^{\# \#} \mathrm{p}$-valores de heterogeneidade $<0,001$. 


\section{ABSTRACT}

Background: This study aims to identify the satisfaction of hypertensive and diabetic users with the care received in primary health care.

Methods: Descriptive analysis and hierarchical models applied to data from the external evaluation of the 2 nd cycle of the Program for Access and Quality Improvement of Primary Care cycle II - PMAQ.

Results: $\mathbf{8 0 . 3 \%}$ out of the 47,927 hypertensive and / or diabetic users in the study $(95 \% \mathrm{Cl} 79.9$ 80.6) were satisfied with the care received. After adjusted analysis, the main factor affecting the outcome was the negative perception of the clinical care received, reducing by $17.0 \%(95 \% \mathrm{Cl} 0.82$ 0.84 ) user satisfaction.

Conclusions: The results reinforce the relevance of investing in actions directed to the health team, providing conditions for improvement in the care offered.

Keywords: Primary health care; Patient satisfaction; Hypertension; Diabetes mellitus; Public health. 